\title{
Esophageal Pathology in Asymptomatic and Symptomatic Patients with Obesity Undergoing Evaluation for Bariatric Surgery
}

\author{
Priya Sharma $^{1}$ (1) $\cdot$ Fady Youssef $^{2} \cdot$ Madeline Greytak $^{2} \cdot$ Ryan Broderick $^{3,4} \cdot$ Garth Jacobsen $^{3,4} \cdot$ Santiago Horgan $^{3,4}$. \\ Rena Yadlapati ${ }^{2}$
}

Received: 8 July 2021 / Accepted: 2 October 2021 / Published online: 20 October 2021

(c) The Author(s) 2021

Keywords Esophageal motility $\cdot$ Gastroesophageal reflux disease $\cdot$ Ambulatory reflux monitoring $\cdot$ High-resolution manometry $\cdot$ Bariatric surgery

Abbreviations
$\begin{array}{ll}\text { GERD } & \text { Gastroesophageal reflux disease } \\ \text { HRM } & \text { High-resolution manometry } \\ \text { BMI } & \text { Body mass index } \\ \text { AET } & \text { Acid exposure time } \\ \text { RYGB } & \text { Roux-en-Y gastric bypass }\end{array}$

\section{Introduction}

Bariatric surgery is the gold standard in achieving long-term weight loss [1]. Esophageal pathology, including gastroesophageal reflux disease (GERD) and esophageal dysmotility, is common after bariatric surgery. However, it is often unclear as to whether pathology exists in obesity prior to surgery. Both anatomic and physiologic processes in obesity can impact esophageal function, which in turn may impact outcomes following surgery [2-5]. Overall, the prevalence and characteristics of esophageal disorders in obesity are not well understood.

Further, the value of esophageal physiologic testing prior to bariatric surgery, such as barium esophagram,

Rena Yadlapati

ryadlapati@health.ucsd.edu

1 Department of Medicine, University of California, La Jolla, San Diego, CA, USA

2 Division of Gastroenterology, Center for Esophageal Diseases, University of California, ACTRI, Building 1W517, 9500 Gilman Drive MC 0956, La Jolla, San Diego, CA 92093, USA

3 Department of Surgery, University of California, La Jolla, San Diego, CA, USA

4 Bariatric and Metabolic Institute, University of California, La Jolla, San Diego, CA, USA reflux monitoring, or high-resolution manometry (HRM), is not well defined or standardized across centers. At our center, the standard protocol requires pre-operative esophageal HRM (Medtronic, Minneapolis, MN) for all patients, whether symptomatic or asymptomatic, and often includes esophagogastroduodenoscopy, ambulatory reflux monitoring, and barium esophagram. Thus, the primary aims of this study were to characterize esophageal physiology in patients with obesity and compare physiologic patterns between patients with and without esophageal symptoms. Based on these findings, we hypothesize our findings will support the utility of HRM prior to bariatric surgery, particularly in patients with symptoms.

\section{Methods}

This retrospective study included adult patients with obesity (body mass index $(\mathrm{BMI}) \geq 35 \mathrm{~kg} / \mathrm{m}^{2}$ ) undergoing preoperative bariatric surgical evaluation at a single tertiary care center between 2/2019 and 2/2020. Symptoms were recorded based on routine standardized patient-reported instruments. Asymptomatic patients were those without dysphagia, heartburn, regurgitation, or non-cardiac chest pain. Symptomatic presentation was defined as reporting at least one of these symptoms. Motility diagnosis was determined per Chicago Classification version 3.0 [6]. Objective GERD was defined as meeting one of the following criteria: (1) acid exposure time (AET) $\geq 4.0 \%$, (2) presence of erosive esophagitis on endoscopy, or (3) confirmed Barrett's esophagus. Primary surgical data collected included the type of weight loss surgery performed: Roux-en-Y gastric bypass (RYGB) or laparoscopic sleeve gastrectomy. 


\section{Results}

A total of 300 adult patients underwent bariatric surgical evaluation with HRM and were included in this analysis: mean age $46.3 \pm 13.6$ years, $226(75.3 \%)$ female, mean BMI $45.2 \pm 8.8 \mathrm{~kg} / \mathrm{m}^{2}$. Of the $300,196(65.3 \%)$ were symptomatic and $104(34.7 \%)$ were asymptomatic (Table 1). Symptom presentation was as follows: $56.3 \%$ heartburn, $28.7 \%$ dysphagia, $27.0 \%$ regurgitation, and $29.3 \%$ noncardiac chest pain.
On HRM, abnormal esophageal motility was seen in $40.3 \%$ of all patients, with $34 \%$ of asymptomatic patients having abnormal motility patterns. Distribution of esophageal motility disorders significantly differed with a greater proportion of ineffective esophageal motility in symptomatic vs asymptomatic patients (36 (18\%) vs $7(7 \%) ; p=0.03)$. The distribution of manometric esophagogastric junction outflow obstruction was similar among symptomatic and asymptomatic patients (21\% vs $21 \%$ ) (Fig. 1). Baseline esophagogastric junction pressure was higher among symptomatic vs asymptomatic patients asymptomatic vs symptomatic $(27.8 \pm 14.5$ vs $31.3 \pm 13.8 \mathrm{mmHg}, p=0.04)$.

Table 1 Baseline characteristics, manometric findings, and objective GERD among asymptomatic and symptomatic patients

\begin{tabular}{|c|c|c|c|}
\hline Variable & Asymptomatic $(n=104)$ & Symptomatic $(n=196)$ & $p$-value \\
\hline Age, years & $43.0 \pm 13.1$ & $48.1 \pm 13.5$ & 0.002 \\
\hline Female gender & $79(76 \%)$ & $147(75 \%)$ & 0.85 \\
\hline BMI, $\mathrm{kg} / \mathrm{m}^{2}$ & $47.7 \pm 8.5$ & $43.9 \pm 8.7$ & $<0.001$ \\
\hline \multicolumn{4}{|l|}{ High-resolution manometry } \\
\hline Hiatal hernia & $23(34 \%)$ & $61(38 \%)$ & 0.64 \\
\hline Hiatal hernia size, $\mathrm{cm}$ & $0.40 \pm 1.11$ & $0.62 \pm 1.42$ & 0.18 \\
\hline EGJ baseline pressure, $\mathrm{mmHg}$ & $31.3 \pm 13.8$ & $27.8 \pm 14.5$ & 0.04 \\
\hline Median IRP, mmHg & $10.69 \pm 6.2$ & $10.04 \pm 7.9$ & 0.47 \\
\hline Mean DCI, mmHg-s-cm & $2257 \pm 1708$ & $1825 \pm 1398$ & 0.02 \\
\hline Mean distal latency, $\mathrm{s}$ & $6.8 \pm 1.6$ & $7.0 \pm 2.4$ & 0.42 \\
\hline$\%$ bolus clearance incomplete & $21 \pm 29$ & $22 \pm 32$ & 0.67 \\
\hline \multicolumn{4}{|l|}{ Objective GERD } \\
\hline Esophageal acid exposure time $(\%$ time $\mathrm{pH}<4.0)$ & $6.8 \pm 1.6(n=4)$ & $7.6 \pm 7.1(n=63)$ & 0.82 \\
\hline Esophageal acid exposure time $>4.0 \%$ & $2(1.9 \%)$ & $40(20.4 \%)$ & $<0.001$ \\
\hline Erosive esophagitis & $1(1.4 \%)(n=73)$ & $19(11.3 \%)(n=168)$ & \\
\hline Barrett's esophagus & $3(4.1 \%)(n=73)$ & $11(6.5 \%)(n=168)$ & \\
\hline $\begin{array}{l}\text { Objective GERD (defined as: AET }>4.0 \% \text {, erosive } \\
\text { esophagitis, and/or Barrett's esophagus) }\end{array}$ & $5(6.8 \%)(n=73)$ & $55(32.4 \%)(n=170)$ & $<0.001$ \\
\hline
\end{tabular}

EGJ, esophagogastric junction; IRP, integrated relaxation pressure; DCI, distal contractile integral; GERD, gastroesophageal reflux disease; AET, acid exposure time. Continuous data presented as mean \pm standard deviation, categorical data presented as $n(\%)$

Fig. 1 Distribution of motility disorders between total, asymptomatic, and symptomatic patients. Esophagogastric junction outflow obstruction (EGJOO); spastic esophageal disorders include distal esophageal spasm (DES) and hypercontractile esophagus; hypomotile esophageal disorders include ineffective esophageal motility (IEM), fragmented peristalsis, and absent contractility

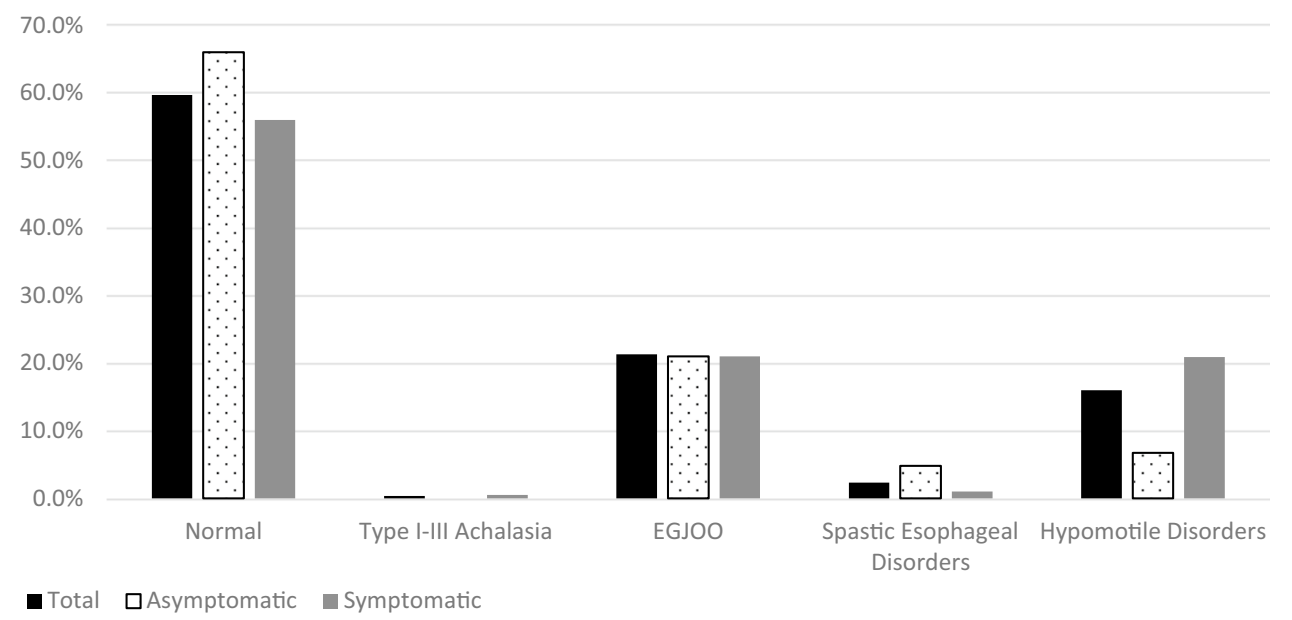


Ability to assess for objective GERD was available for $243(81 \%)$ patients: $170(70.0 \%)$ symptomatic and 73 (30.0\%) asymptomatic. Among the 243 patients, $32.4 \%$ of symptomatic vs $6.8 \%$ of asymptomatic patients had evidence of objective GERD, providing 5.3 times higher odds of objective GERD in symptomatic patients compared to asymptomatic patients $(95 \%$ CI $1.45,20.0 ; p=0.01)$. Of those who underwent bariatric surgery, symptomatic patients were more likely to receive RYGB compared to asymptomatic patients (33\% vs 7\%) and less likely to receive sleeve gastrectomy ( $67 \%$ vs $93 \% ; p<0.01)$.

\section{Conclusion}

Esophageal dysmotility and reflux are common in obesity. Symptoms of heartburn, regurgitation, dysphagia, and noncardiac chest pain may suggest abnormal esophageal motility and/or esophageal reflux. These data suggest a role of HRM and reflux monitoring in patients with esophageal symptoms including obstructive and typical reflux symptoms prior to bariatric surgery, as detection of dysmotility and objective GERD may influence preoperative course.

Acknowledgements Study design: PS, FY, MG, RB, GJ, SH, RY; data collection: PS, FY, MG, RY; analysis and interpretation of data: PS, FY, RY; drafting of manuscript: PS, FY, RY; critical revision of the manuscript for important intellectual content: PS, FY, MG, RB, GJ, SH, RY; finalization of manuscript: PS, FY, MG, RB, GJ, SH, RY

Funding RY is supported by NIH K23 DK125266 (PI: Yadlapati).

\section{Declarations}

Conflict of Interest PS, FY, MG, RB, GJ, SH: None; RY: Institutional Consulting Agreement: Medtronic, Ironwood Pharmaceuticals, Di- versatek; Consultant: Phathom Pharmaceuticals; Research support: Ironwood Pharmaceuticals; Advisory Board with Stock Options: RJS Mediagnostix.

Open Access This article is licensed under a Creative Commons Attribution 4.0 International License, which permits use, sharing, adaptation, distribution and reproduction in any medium or format, as long as you give appropriate credit to the original author(s) and the source, provide a link to the Creative Commons licence, and indicate if changes were made. The images or other third party material in this article are included in the article's Creative Commons licence, unless indicated otherwise in a credit line to the material. If material is not included in the article's Creative Commons licence and your intended use is not permitted by statutory regulation or exceeds the permitted use, you will need to obtain permission directly from the copyright holder. To view a copy of this licence, visit http://creativecommons.org/licenses/by/4.0/.

\section{References}

1. 1.Buchwald, H., et al., Bariatric surgery: a systematic review and meta-analysis. JAMA, 2004. 292(14): p. 1724-37.

2. 2.Hong, D., et al., Manometric abnormalities and gastroesophageal reflux disease in the morbidly obese. Obes Surg, 2004. 14(6): p. 744-9.

3. 3.Barak, N., et al., Gastro-oesophageal reflux disease in obesity: pathophysiological and therapeutic considerations. Obes Rev, 2002. 3(1): p. 9-15.

4. 4.Koppman, J.S., et al., Esophageal motility disorders in the morbidly obese population. Surg Endosc, 2007. 21(5): p. 761-4.

5. 5.Suter, M., et al., Gastro-esophageal reflux and esophageal motility disorders in morbidly obese patients. Obes Surg, 2004. 14(7): p. 959-66.

6. 6.Kahrilas, P.J., et al., The Chicago Classification of esophageal motility disorders, v3.0. Neurogastroenterol Motil, 2015. 27(2): p. 160-74.

Publisher's Note Springer Nature remains neutral with regard to jurisdictional claims in published maps and institutional affiliations. 